\title{
TROJANS AND COMETS OF THE JUPITER GROUP
}

\author{
EUGENE RABE \\ Cincinnati Observatory
}

In a recent paper (Rabe, 1970), I suggested that at least some comets of the Jupiter group may have originated from the relatively dense Trojan clouds that, according to a recent survey by the van Houtens and Gehrels (1970), seem to be associated with the equilateral points $L_{4}$ and $L_{5}$ of the Jupiter orbit. This suggestion was inspired by the finding that the periodic comet SlaughterBurnham has been captured (or perhaps recaptured) into unstable or temporary "Trojan" librations lasting approximately $2500 \mathrm{yr}$ and by the circumstance that the Jacobi "constants" of most Jupiter group comets have values between 3.0 and 2.5 , or just in that range which would also be occupied by all known and unknown Trojans associated with Jupiter in stable or unstable librations involving heliocentric eccentricities up to about 0.5 and inclinations as large as $30^{\circ}$. Moreover, it is well known that nearly all comets of the Jupiter group are able to approach Jupiter rather closely, thus providing the possibility for such drastic orbital changes as temporary capture into, or escape from, librational motion of the Trojan type. All, presumably stable, orbits of actual Trojan planets presently known have eccentricities $e$ not exceeding 0.15 , but we also know that stable short-period librations with much larger $e$ values do exist in the restricted Sun-Jupiter problem, so that even in the real, nonrestricted situation there should be a possibility for corresponding librations with these more substantial short-period components. These librations may be unstable but may have long lifetimes nevertheless. The case of $\mathrm{P} /$ Slaughter-Burnham has proved that such motions are indeed possible, even with an $e$ as large as 0.52 .

The Jacobi integral is valid only in the restricted three-body problem, but the experience from many numerical integrations indicates that in the real Sun-Jupiter elliptic problem, with $e^{\prime} \sim 0.05$, the Jacobi "constant" $C$ from the approximating Tisserand criterion,

$$
C=\frac{1}{a}+2 \sqrt{a} \cos \phi \cos I
$$

with

$$
\cos I=\cos i \cos i^{\prime}+\sin i \sin i^{\prime} \cos \left(\Omega-\Omega^{\prime}\right)
$$


tends to vary only within relatively narrow limits, as long as the osculating elements used in evaluating equation (1) do not belong to some moment at which the small body in question is inside of Jupiter's gravitational "sphere of action." In equations (1) and (2), $a$ is the semimajor axis of the Trojan's or comet's heliocentric orbit expressed in units of the mean Sun-Jupiter distance, $\phi$ is related to $e$ through $e=\sin \phi$, and $I$ denotes the orbital inclination relative to the Jupiter orbit as computed from the respective ecliptical inclinations $i$ and $i^{\prime}$ and nodes $\Omega$ and $\Omega^{\prime}$ according to equation (2). The prime symbol denotes the elements of Jupiter.

For the overwhelming majority of all minor planets, $C>3$ and $a<1$. To the exceptions with $C<3$ belong all the known Trojans (because of their small deviations from $a_{0}=a^{\prime}=1$ in combination with nonvanishing $I$ and $e$ ), several members of the Hilda family with their also relatively large $a$ values of the order of 0.8 , and some asteroids with smaller values of $a$ but with such exceptionally large values of $e$ or/and $I$, as to enable the second term of the right-hand side of equation (1) to become sufficiently small. If the auxiliary angle $\gamma$ is introduced through

$$
\cos \gamma=\cos \phi \cos I
$$

equation (1) can be solved to express $\cos \gamma$ as a function of the nearly constant $C$ and of the variable $a$. Assuming that at some time the value $a_{0}=1$ can be attained by $a$, in consequence of Jupiter's perturbing action, the resulting function $\cos \gamma$ reduces to

$$
\cos \gamma_{0}=\frac{1}{2}(C-1)
$$

This equation contains the well-known statement that the attainment of $a_{0}=1$, and thus a "crossover" from $a<1$ to $a>1$ or vice versa, is impossible if $C>3$. Clearly, $C>3$ leads to $\cos \gamma_{0}>1$ and thus to either $\cos \phi>1$ or $\cos$ $I>1$, if not to both inequalities togethci. Actually, because terms of the order of Jupiter's mass, $\mu \sim 0.001$, have been neglected in equation (1), even if applied to the restricted problem with $e^{\prime}=0$, the subsequent equation (4) proves the impossibility of crossovers only for $C$ values that exceed 3.000 by amounts larger than some quantity of order $\mu$. Conservatively, one may require $C>3.01$. Considering also Jupiter's orbital eccentricity $e^{\prime} \sim 0.05$, however, in conjunction with the resulting slight variability of $C$ (as evidenced in many relevant numerical integrations), the critical $C$ limit for the possible occurrence of $a_{0}=1$ has to be increased even more. Indications are that the effective limit (in the absence of significant perturbations from other major planets) lies near $C=3.03$. It also appears that for any asteroids or comets within $3.00<C<3.03$, any crossovers would tend to happen through temporary capture into satellite rather than Trojan status, as evidenced by the $C$ values that one finds for the many sets of elements $a, e$, and $I$ obtained by Hunter (1967) in his work on satellite/asteroid transfers. For any comets with 
$C$ values close to 3.00 , the possibility of temporary satellite capture must definitely be considered together with that of temporary Trojan-type librations. For nearly all Jupiter group comets, however, one finds $C \leqslant 2.97$; therefore, satellite captures become rather unlikely. Such $C$ values fall into the range typical for Trojan librations (as low as $C=2.673$ for 1208 Troilus, among the known Trojan planets). Comets, however, may pass through $a_{0}=1$ in most cases, of course, without getting caught into temporary oscillations of $a$ within the narrow boundaries $0.95 \leqslant a \leqslant 1.05$ required for Trojan-type librations (Rabe, 1970).

Because the Jupiter group comets as well as the Trojans are able, practically by definition, to experience crossovers through $a_{0}=1$, it should, of course, be expected that the $C$ distributions of both groups will overlap. On the other hand, all known Trojans vary their osculating $a$ values within the rather narrow limits $0.97 \leqslant a \leqslant 1.03$, whereas the Jupiter comets attain $a$ values as small as $0.44(\mathrm{P} /$ Encke) and as large as $1.38(\mathrm{P} /$ Oterma). For the eccentricities, the observed upper limit $e=0.15$ for the Trojans has already been mentioned, whereas the comets considered here have $e$ values up to 0.85 . Only the inclinations $I$ have similar distributions in both groups, with a very few being somewhat larger than $30^{\circ}$ in each category. On the basis of their significantly different $a$ and $e$ distributions, however, it would not be surprising to find rather different $C$ distributions, too, with a similarly narrow overlap as in $a$ and $e$. As repeatedly mentioned, though, there is an extensive overlap of the two $C$ distributions, suggesting a close dynamical affinity of the two groups of bodies. The principal purpose of this paper is the computation and presentation of a sufficiently large number of individual $C$ values, as well as of some related quantities that are of interest with regard to possible conjectures concerning a dynamical relationship between Trojans and Jupiter group comets.

For the 15 numbered Trojan planets, the elements needed in equations (1) and (2) have been taken from the Leningrad Ephemeris volume for 1971, except for those of the not yet listed newest member 1749 Telamon, which are given in Minor Planet Circular 3019. For 38 comets of the Jupiter group rather approximate but for the present purpose sufficiently accurate elements were taken from Marsden's (1967) tabulation of such comets with $a$ values presently $(\sim 1965)$ inside Jupiter's $a^{\prime}=1$. Three other comets were added, without any at tempt to achieve completeness: P/Oterma (Astron. J. 66, 248), P/Schwassmann-Wachmann 1 (Astron. J. 66, 268), and P/Slaughter-Burnham (Acta Astron. 18, 419). Finally, the quite exceptional minor planet 944 Hidalgo was also included here for the sake of comparison. For the total of 57 objects, the computed $C$ values are listed in decreasing order in table I, together with the "crossover parameter" $\gamma_{0}$ from equation (4). Of the basic elements $a, e$, and $I$, only $e$ has been listed, under the heading $e_{1}$, so that it may be compared with the $e_{0}$ given in the last column, which is the largest possible value of $e$ at crossover in connection with $a=1$. Because equation (3) has to be satisfied also when $\gamma=\gamma_{0}$, this maximum $e_{0}$ would occur only in conjunction with $I=0$, whereas the also possible maximum $I(a=1, \phi=0)=\gamma_{0}$ would 
TABLE I.-The Jacobi Constant $\mathrm{C}$ and Related Quantities for Trojans and Selected Jupiter Group Comets

\begin{tabular}{|c|c|c|c|c|}
\hline Comet or Trojan & $C$ & $\begin{array}{c}\gamma_{0} \\
(a=1)\end{array}$ & $e_{1}$ & $\begin{array}{c}e_{0} \\
(a=1, I=0)\end{array}$ \\
\hline Oterma & 3.00 & $0^{\circ}$ & 0.25 & 0.00 \\
\hline Schwassmann-Wachmann 2 & 3.00 & 0 & .38 & .00 \\
\hline 1143 Odysseus & 2.986 & - & .093 & - \\
\hline 1647 Menelaus & 2.986 & - & .028 & - \\
\hline 659 Nestor & 2.980 & - & .110 & - \\
\hline 1749 Telamon & 2.974 & - & .111 & - \\
\hline Encke & 2.97 & 9 & .85 & .16 \\
\hline Tempel 1 & 2.97 & 10 & .52 & .17 \\
\hline Schwassmann-Wachmann 1 & 2.97 & 10 & .14 & .17 \\
\hline 1173 Anchises & 2.961 & - & .136 & - \\
\hline Tempel 2 & 2.96 & 11 & .55 & .19 \\
\hline 884 Priamus & 2.955 & - & .120 & - \\
\hline Whipple & 2.95 & 13 & .35 & .23 \\
\hline Johnson & 2.94 & 14 & .38 & .24 \\
\hline 588 Achilles & 2.939 & - & .148 & - \\
\hline Neujmin 2 & 2.92 & 16 & .58 & .28 \\
\hline de Vico-Swift & 2.91 & 17 & .52 & .29 \\
\hline Reinmuth 2 & 2.91 & 17 & .46 & .29 \\
\hline Brooks 2 & 2.90 & 19 & .50 & .32 \\
\hline 1172 Äneas & 2.895 & - & .102 & - \\
\hline 624 Hektor & 2.892 & - & .025 & - \\
\hline Ashbrook-Jackson & 2.89 & 19 & .40 & .33 \\
\hline $1404 \mathrm{Ajax}$ & 2.880 & - & .113 & - \\
\hline Forbes & 2.87 & 21 & .55 & .35 \\
\hline 1437 Diomedes & 2.859 & - & .046 & - \\
\hline Kopff & 2.85 & 22 & .56 & .37 \\
\hline Holmes & 2.85 & 22 & .38 & .38 \\
\hline 617 Patroclus & 2.844 & - & .141 & - \\
\hline 911 Agamemnon & 2.843 & - & .067 & - \\
\hline Tempel-Swift & 2.84 & 23 & .54 & .40 \\
\hline Wirtanen & 2.84 & 23 & .54 & .40 \\
\hline Reinmuth 1 & 2.84 & 23 & .49 & .40 \\
\hline Tuttle-Giacobini-Kresák & 2.83 & 24 & .64 & .41 \\
\hline Grigg-Skjellerup & 2.82 & 24 & .66 & .41 \\
\hline Harrington & 2.81 & 25 & .56 & .42 \\
\hline Wolf-Harrington & 2.79 & 26 & .54 & .44 \\
\hline Harrington-Abell & 2.79 & 27 & .52 & .45 \\
\hline 1583 Antilochus & 2.745 & - & .054 & - \\
\hline Daniel & 2.74 & 29 & .55 & .49 \\
\hline Faye & 2.74 & 30 & .58 & .49 \\
\hline d'Arrest & 2.73 & 30 & .61 & .50 \\
\hline Arend-Rigaux & 2.72 & 30 & .60 & .51 \\
\hline Wolf & 2.72 & 31 & .39 & .51 \\
\hline Slaughter-Burnham & 2.71 & 31 & .50 & .52 \\
\hline Arend & 2.70 & 32 & .53 & .53 \\
\hline Pons-Winnecke & 2.69 & 32 & .64 & .54 \\
\hline 1208 Troilus & 2.673 & - & .092 & - \\
\hline
\end{tabular}


TABLE I.-The Jacobi Constant $\mathrm{C}$ and Related Quantities for Trojans and Selected Jupiter Group Comets-Concluded

\begin{tabular}{l|c|c|c|c}
\hline \multicolumn{1}{c|}{ Comet or Trojan } & $C$ & $\begin{array}{c}\gamma_{0} \\
(a=1)\end{array}$ & $e_{1}$ & $\begin{array}{c}e_{0} \\
(a=1, I=0)\end{array}$ \\
\hline Perrine-Mrkos & 2.67 & 33 & .64 & .55 \\
Comas Solá & 2.67 & 34 & .58 & .55 \\
Finlay & 2.63 & 35 & .70 & .58 \\
Borrelly & 2.60 & 37 & .60 & .60 \\
Honda-Mrkos-Pajdušáková & 2.56 & 39 & .82 & .63 \\
Biela & 2.54 & 40 & .76 & .64 \\
Schaumasse & 2.49 & 42 & .71 & .66 \\
Brorsen & 2.47 & 43 & .83 & .68 \\
Giacobini-Zinner & 2.46 & 43 & .73 & .68 \\
944 Hidalgo (non-Trojan & 2.07 & 58 & .65 & .85 \\
$\quad$ minor planet for & & & & \\
\multicolumn{2}{c}{ comparison) } & & &
\end{tabular}

require $e=0$. It is well known that $I$ as well as $e$ may undergo large variations during close approaches to Jupiter, so that the possibility of a near-zero inclination $I$ after some approach cannot be excluded. Normally, though, $I$ will not become very small; and, consequently, the related crossover eccentricity $e$ satisfying equation (3) will be smaller than the listed maximum $e_{0}$. No $e_{0}$ values have been computed for the 15 numbered Trojans, because it may be assumed that they move in stable libration orbits and do not experience close approaches to Jupiter. We know that their $e$ and $I$ values vary only rather moderately during the small long-period oscillations of $a$, so that the formal computation of $e_{0}$ for $I=0$ would make no physical sense. If they would be computed and given, they would fall right into the systematic trend of this last column in table I, because the listing is in order of $C$, and $e_{0}$ and $\gamma_{0}$ are functions of $C$ alone. To facilitate the separate recognition of Trojans and comets in table I, the $\gamma_{0}$ values of the Trojans have been omitted as well.

It is seen from table I that even the numbered and presumably stable Trojans have $C$ values that extensively overlap those of the Jupiter comets. It is very likely that most of the at least several hundred additional Trojans (van Houten et al., 1970) fall into about the same range, $2.67 \leqslant C<3.00$, simply because of their probably stable association with the equilateral points. Of the 41 comets in table I, 34, or 83 percent, also have $C$ values between 2.67 and 3.00. For those contemplated unstable or escaped Trojans with initial $e$ values exceeding 0.15 , the related $C$ values could easily be as small as 2.5 ; therefore, there could be a complete overlap of the ranges of $C$ values for the comets and Trojans. Of particular interest are the $e_{0}$ values of the 41 comets. Most of them are much smaller than the associated $e_{1}$ values; therefore, in connection with the suggested Trojan origin of such comets, the related values of $e$ for $a=1$ should be in quite reasonable agreement with the $e$ range that is permissible by 
dynamical considerations for librational motions of the Trojan type. First, the actual crossover values of $e$ will normally be smaller than the maximum $e_{0}$; and, second, on the theoretical side, we know that in the restricted Sun-Jupiter case, even stable librations of short period may involve $e$ values near 0.64 . In the one known case of temporary librations, for P/Slaughter-Burnham, such motion actually occurs with $e \sim 0.52$.

When the possibility of Trojan origin for some Jupiter group comets was first suggested (Rabe, 1970), the detailed features of the reverse event of capture into libration for $\mathrm{P} /$ Slaughter-Burnham were interpreted as indicating a rather small probability of such capture for any given comet because, in most cases, the large perturbations in $a$ during the required Jupiter approach will tend to overshoot the apparently necessary entry conditions. Such probability considerations have no bearing on the contemplated escapes from librations of an unstable nature. The only requirement seems to be that the original Trojan clouds had to be large enough to permit the formation and growth of condensations even near the fringes of librational stability.

When Oort (1950) discussed the proposed existence of a very distant cloud of comets surrounding the solar system, he suggested that these bodies might actually be unstable escapees from the original minor planet belt between Mars and Jupiter. It appears now that, at least for the Jupiter comets, the escape from the two Trojan clouds provides a much simpler and more direct mechanism of asteroid transfer into cometary motion, without the need of moving these bodies first to the remote fringes of the solar system, and of then recapturing them in a complicated chain of dynamical events. It should be noted again, as in Rabe (1970), that the similarity of the anomalous distributions of the perihelion longitudes of the Trojans and of the Jupiter group comets lends further support to their proposed common origin. Also, the complete absence of retrograde orbits would automatically be accounted for by such an origin of these comets. The relatively large masses of some Trojans can no longer be considered as an argument against a common origin, because the van Houtens and Gehrels (1970) have found that the frequency of the Trojans increases greatly with decreasing magnitude.

\section{REFERENCES}

Houten, C. J. van, Houten-Groeneveld, I. van, and Gehrels, T. 1970, Minor Planets and Related Objects. V. The Density of Trojans Near the Preceding Lagrangian Point. Astron. J. 75, 659-662.

Hunter, R. B. 1967, Motions of Satellites and Asteroids Under the Influence of Jupiter and the Sun. II. Asteroid Orbits Close to Jupiter. Mon. Notic. Roy. Astron. Soc. 136, 267-277.

Marsden, B. G. 1967, One Hundred Periodic Comets. Science 155, 1207-1213.

Oort, J. H. 1950, The Structure of the Cloud of Comets Surrounding the Solar System, and a Hypothesis Concerning Its Origin. Bull. Astron. Inst. Neth. 11, 91-110.

Rabe, E. 1970, Orbital Characteristics of Comets Passing Through the 1:1 Commensurability With Jupiter. Proc. IAU Symp. no. 45, Motion, Orbit Evolution and Origin of Comets. Leningrad. 